\title{
On the Effect of Correlation in IRS-Aided SWIPT Networks
}

\author{
Constantinos Psomas and Ioannis Krikidis \\ Department of Electrical and Computer Engineering, University of Cyprus, Cyprus \\ Email: \{psomas, krikidis\}@ucy.ac.cy
}

\begin{abstract}
In this work, we focus on the effect of correlation between the elements of an intelligent reflecting surface, in the context of simultaneous wireless information and power transfer (SWIPT). The two extreme cases are considered, that is, when the elements are uncorrelated (independent) and when the elements are fully correlated. For each case, we study the performance of employing random phase shifts as well as with no phase shifts. Closed-form analytical expression are derived for the outage probability and the average harvested energy. When the elements are uncorrelated, random phase shifts provide the same performance as having no phase shifts. However, we show that the performance of correlated elements attains significant gains when there are no phase shifts and exceeds the performance of the uncorrelated case.
\end{abstract}

Index Terms-Intelligent reflecting surfaces, correlation, SWIPT, outage probability.

\section{INTRODUCTION}

Intelligent reflecting surfaces (IRS) is a technology that has recently received notable consideration by the research community [1]. The reconfigurable nature of this technology has the potential of improving both coverage and energy efficiency through its elements, which reflect the received signal by rotating its phase. Moreover, the fact that an IRS can be embedded on a flat surface, makes it an attractive solution for indoor and outdoor applications. The benefits of IRSs have been shown for numerous communication scenarios and technologies [2]-[5] as well as in the context of simultaneous wireless information and power transfer (SWIPT) [6]. In SWIPT systems, the transmitted signal is used to convey both information and energy. Specifically, information decoding and energy harvesting is achieved at the receiver with the employment of a practical scheme such as time-switching or power-splitting (PS) [7]. Thus, the implementation of SWIPT over an IRS, can provide significant gains to both information decoding and energy harvesting.

Since an IRS consists of multiple adjacent elements, it follows that the elements exhibit a certain spatial correlation between them. Therefore, in this work, we focus on the effect of channel correlation between the elements of an IRS in an IRS-aided SWIPT network. We consider the two extreme cases, i.e. the elements are mutually uncorrelated (independent) and mutually fully correlated. As the phase shifts are not affected

This work was co-funded by the European Regional Development Fund and the Republic of Cyprus through the Research and Innovation Foundation, under the projects POST-DOC/0916/0256 (IMPULSE) and EXCELLENCE/0918/0377 (PRIME). This work has also received funding from the European Research Council (ERC) under the European Union's Horizon 2020 research and innovation programme (Grant agreement No. 819819). by the correlation, we study the achieved performance with the employment of random phase shifts and without phase shifts. Closed-form analytical expression are derived for the information outage probability, the energy outage probability and the average harvested energy. In the case where the elements are uncorrelated, the performance is unaffected, regardless of having random or no phase shifts. On the other hand, for the correlated case, we show that there are significant benefits when there are no phase shifts and the uncorrelated case is outperformed.

\section{System Model}

We consider a simple topology, consisting of a transmitter, a receiver and an IRS located between them. The transmitter and the receiver have a single antenna and the IRS is equipped with $M$ reflecting elements. It is assumed that a direct link between the transmitter and the receiver is not available (e.g., due to heavy shadowing) [2]. All wireless links are assumed to exhibit Rayleigh fading. We define by $h_{i}$ the channel coefficient between the transmitter and the $i$-th IRS element, and by $g_{i}$ the channel coefficient between the $i$-th element and the receiver; these are assumed to be distributed according to a complex Gaussian distribution with zero mean and unit variance, i.e. $h_{i} \sim \mathcal{C N}(0,1)$ and $g_{i} \sim \mathcal{C N}(0,1)$.

Time is slotted and, at each time slot, the $i$-th element shifts the phase of the incident signal to a certain angle $\phi_{i} \in[0,2 \pi)$. Then, the received signal at the receiver is

$$
r=\sqrt{P_{t}} \mathbf{h}^{T} \mathbf{\Phi g} x+n,
$$

where $P_{t}$ is the transmit power, $x$ is the transmitted symbol, $\mathbf{h}=\left[\begin{array}{llll}h_{1} & h_{2} & \ldots & h_{M}\end{array}\right]^{T}, \mathbf{g}=\left[\begin{array}{llll}g_{1} & g_{2} & \ldots & g_{M}\end{array}\right]^{T}, n \sim \mathcal{C N}\left(0, \sigma^{2}\right)$ is an additive white Gaussian noise (AWGN) with variance $\sigma^{2}$, and

$$
\mathbf{\Phi}=\operatorname{diag}\left[\exp \left(\jmath \phi_{1}\right) \exp \left(\jmath \phi_{2}\right) \ldots \exp \left(\jmath \phi_{M}\right)\right]
$$

is the diagonal matrix containing the phase shift variables. As such, the channel gain achieved by the $M$ elements is

$$
H=\left|\sum_{i=1}^{M} h_{i} g_{i} \exp \left(\jmath \phi_{i}\right)\right|^{2} .
$$

The receiver has SWIPT capabilities and splits the power of the received signal into two parts; one part is converted to baseband for information decoding and the other is directed to the rectenna for energy harvesting [7]. Let $0<\rho \leq 1$ denote the power-splitting parameter, i.e. $100 \rho \%$ of the received 
power is used for decoding. The additional circuit noise formed during the RF to baseband conversion phase is modeled as an AWGN with zero mean and variance $\sigma_{C}^{2}$. Therefore, the achieved instantaneous SNR at the receiver is

$$
\gamma=\frac{\rho P_{t} H}{\rho \sigma^{2}+\sigma_{C}^{2}}
$$

where $H$ is given by (3).

Moreover, as $100(1-\rho) \%$ of the received signal is passed to the rectifier, the instantaneous harvested energy is modeled by the following quadratic polynomial [7]

$$
E=(1-\rho)\left(P_{t} H+P_{t}^{2} H^{2}\right) .
$$

Note that any RF energy harvesting from the AWGN noise is considered to be negligible.

\section{IRS-AIDED SWIPT NETWORK}

To evaluate the effect of correlation, we consider three metrics: the information outage probability, the energy outage probability and the average harvested energy.

Let $\tau$ be a non-negative pre-defined rate threshold. Then,

$$
\Pi_{I}(\tau)=\mathbb{P}\left\{\log _{2}(1+\gamma)<\tau\right\},
$$

defines the information outage probability. Similarly, the energy outage probability is given by

$$
\Pi_{E}(\epsilon)=\mathbb{P}\left\{(1-\rho)\left(P_{t} H+P_{t}^{2} H^{2}\right)<\epsilon\right\},
$$

where $\epsilon$ is a non-negative pre-defined energy threshold. Finally, we have

$$
\mathcal{E}=\mathbb{E}_{H}\left\{(1-\rho)\left(P_{t} H+P_{t}^{2} H^{2}\right)\right\},
$$

which provides the average harvested energy. Each metric is evaluated analytically for the uncorrelated and correlated cases, given in the following subsections.

\section{A. Uncorrelated Case}

In this case, the channel gain is given by (3). We provide the following proposition for the information outage probability without proof, as it follows directly from [5].

Proposition 1. In the uncorrelated case, the outage probability achieved by an IRS with $M$ elements is given by

$$
\Pi_{I}^{u}(\tau)=1-\frac{2 \Theta^{\frac{M}{2}}}{\Gamma(M)} K_{M}(2 \sqrt{\Theta})
$$

where

$$
\Theta=\frac{\left(2^{\tau}-1\right)\left(\rho \sigma^{2}+\sigma_{c}^{2}\right)}{\rho P_{t}} .
$$

Based on the above proposition, we can state the following corollary, where $\Theta$ is obtained by taking the root of the quadratic polynomial in (5).

Corollary 1. The energy outage probability $\Pi_{E}^{u}(\epsilon)$, in the uncorrelated case, is given by Proposition 1 with

$$
\Theta=\frac{\sqrt{1+4 \epsilon /(1-\rho)}-1}{2 P_{t}} .
$$

Finally, the average harvested energy is obtained from $\mathbb{E}\{H\}=M$ and $\mathbb{E}\left\{H^{2}\right\}=2 M(M+1)$.

Proposition 2. The average harvested energy for the uncorrelated case is

$$
\mathcal{E}_{u}=(1-\rho) M P_{t}\left(1+2 P_{t}(M+1)\right) .
$$

Note that the first term in (12) corresponds to the linear model. Moreover, all of the above results also hold for the case with no phase shifts.

\section{B. Correlated Case}

In the case of correlated elements with random phase shifts, the channel gain is $H=|h|^{2}|g|^{2}\left|\sum_{i=1}^{M} \exp \left(\jmath \phi_{i}\right)\right|^{2}$. Then, we can state the following results.

Proposition 3. In the correlated case with random phase shifts, the outage probability achieved by an IRS with $M$ elements is given by

$$
\Pi_{I}^{c}(\tau)=1-\frac{2}{M} \int_{0}^{\infty} \sqrt{\frac{\Theta}{\theta}} \exp \left(-\frac{\theta}{M}\right) K_{1}\left(2 \sqrt{\frac{\Theta}{\theta}}\right) d \theta
$$

where $\Theta$ is given by (10).

Proof. See Appendix.

The next corollary follows from Proposition 3, where $\Theta$ is obtained by taking the root of the quadratic polynomial in (5).

Corollary 2. The energy outage probability $\Pi_{E}^{c}(\epsilon)$, in the correlated case with random phase shifts, is given by Proposition 3 with $\Theta$ given by (11).

Proposition 4. The average harvested energy for the correlated case with random phase shifts is

$$
\mathcal{E}_{c}=(1-\rho) M P_{t}\left(1+4 P_{t}(2 M-1)\right) .
$$

Proof. In this case, $\mathbb{E}\{H\}=M$ and $\mathbb{E}\left\{H^{2}\right\}=4 M(2 M-1)$, and the result follows.

By comparing (12) to (14), it is easy to deduce that $\mathcal{E}_{c}>$ $\mathcal{E}_{u}$ for $M>1$. It is important to note that the linear energy harvesting model would not have provided this observation. Finally, we provide the analytical expressions for the correlated case with no phase shifts; in this case, $H=M^{2}|h|^{2}|g|^{2}$. The proofs follow similarly with the previous statements and so are omitted.

Proposition 5. In the correlated case with no phase shifts, the outage probability achieved by an IRS with $M$ elements is given by

$$
\Pi_{I}^{c n}(\tau)=1-\frac{2}{M} \sqrt{\Theta} K_{1}\left(2 \frac{\sqrt{\Theta}}{M}\right),
$$

where $\Theta$ is given by (10).

Corollary 3. The energy outage probability $\Pi_{E}^{c n}(\epsilon)$, in the correlated case with no phase shifts, is given by Proposition 5 with $\Theta$ given by (11). 

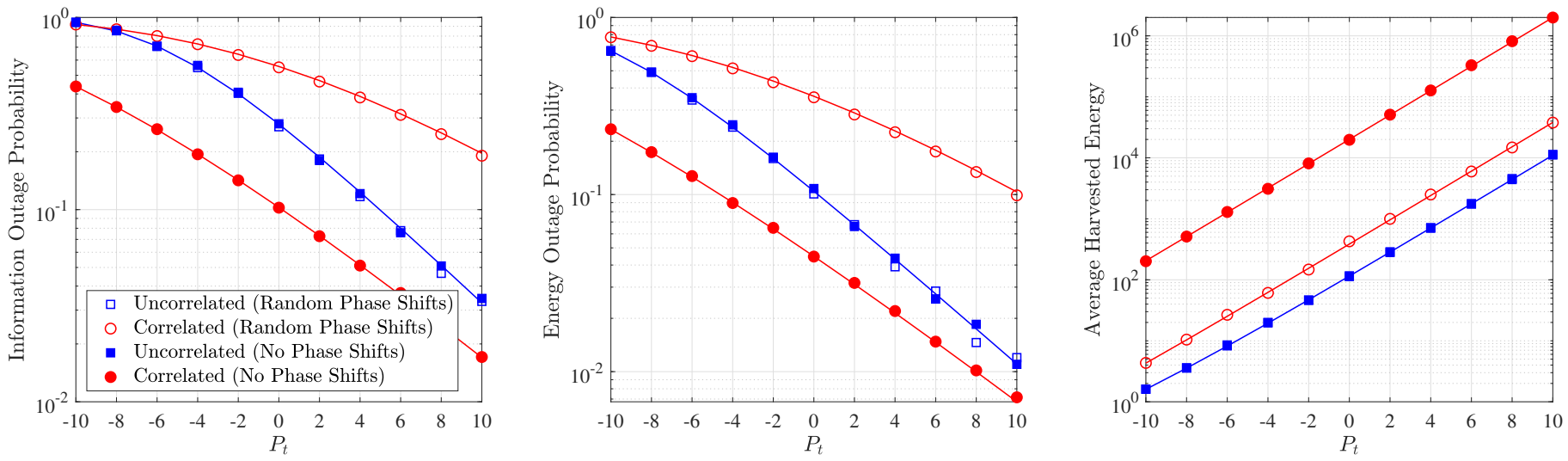

Fig. 1. Information outage probability, energy outage probability and average harvested energy vs $P_{t}$.

Proposition 6. The average harvested energy for the correlated case with no phase shifts is

$$
\mathcal{E}_{c n}=(1-\rho) M^{2} P_{t}\left(1+4 P_{t} M^{2}\right) .
$$

It is clear that $\mathcal{E}_{c n}>\mathcal{E}_{c}>\mathcal{E}_{u}$ for $M>1$.

\section{Numerical Results}

We verify the above analytical framework with Monte Carlo simulations. For the sake of presentation, we consider the following parameters: $\rho=0.5, M=10, \tau=1 \mathrm{bpcu}, \epsilon=1 \mathrm{~J}$, $\sigma^{2}=0 \mathrm{~dB}$ and $\sigma_{C}^{2}=0 \mathrm{~dB}$.

In Fig. 1, the sub-figures from left to right illustrate the information outage probability, the energy outage probability and the average harvested energy in terms of the transmit power $P_{t}$. As expected, the increase in transmit power benefits all cases and metrics. If random phase shifts are employed, the uncorrelated case outperforms the correlated in terms of outage (both information and energy). However, in terms of average harvested energy, the correlated case provides higher values. This implies that, in the case of correlation, the system should prefer a long term energy harvesting strategy. On the other hand, in the case of no correlation, the system should follow an instantaneous policy. Now, if phase shifts are not employed, the correlated case performs significantly better in all three metrics. This means that phase shifting is a degrading factor when the elements are correlated. Moreover, note that the uncorrelated case is independent of the phase shift values as pointed it out in the previous section. Finally, the markers (simulation results) perfectly match the lines (theoretical results), which validates our analytical framework.

\section{CONCLUSION}

In this work, we focused on the effect of correlation between the elements of an IRS in the context of SWIPT. Two cases were considered, uncorrelated and fully correlated. We showed that when the phases are randomly shifted, the uncorrelated case provides the best performance in terms of information and energy outage but is outperformed in terms of average harvested energy. However, when there are no phase shifts, the correlated case outperforms the uncorrelated one in all metrics. Future directions include modeling the correlation as a function of the distance between two elements.

\section{APPENDIX}

Let $\theta=\left|\sum_{i=1}^{M} \exp \left(\jmath \phi_{i}\right)\right|^{2}$. Then, by employing the central limit theorem, it is easy to deduce that $\theta$ is exponentially distributed with parameter $1 / M$. In addition, the cumulative distribution function of $|h|^{2}|g|^{2}$ is [8]

$$
F_{|h|^{2}|g|^{2}}(z)=1-2 \sqrt{z} K_{1}(2 \sqrt{z}) .
$$

Therefore, from (6) we have

$$
\Pi_{I}^{c}(\tau)=\mathbb{E}_{\theta}\left\{1-2 \sqrt{\frac{\Theta}{\theta}} K_{1}\left(2 \sqrt{\frac{\Theta}{\theta}}\right)\right\},
$$

where $\Theta$ is given by (10). By un-conditioning with $f_{\theta}(\theta)=$ $\exp (-\theta / M) / M$, the proposition is proved.

\section{REFERENCES}

[1] L. Dai, et al., "Reconfigurable intelligent surface-based wireless communication: Antenna design, prototyping and experimental results," IEEE Access, vol. 8, pp. 45913-45923, Mar. 2020.

[2] Q. Nadeem, A. Kammoun, A. Chaaban, M. Debbah, M.-S Alouini, "Asymptotic max-min SINR analysis of reconfigurable intelligent surface assisted MISO systems," [Online]. Available: https://arxiv.org/abs/1903.08127

[3] Y. Han, W. Tang, S. Jin, C.-K. Wen, and X. Ma, "Large intelligent surfaceassisted wireless communication exploiting statistical CSI," IEEE Trans. Veh. Tech., vol. 68, no. 8, pp. 8238-8242, June 2019.

[4] M. Cui, G. Zhang, and R. Zhang, "Secure wireless communication via intelligent reflecting surface," IEEE Wireless Commun. Lett., vol. 8, no. 5, pp. 1410-1414, May 2019.

[5] C. Psomas and I. Krikidis, "Low-complexity random rotationbased schemes for intelligent reflecting surfaces," [Online] Available: https://arxiv.org/abs/1912.10347

[6] C. Pan, H. Ren, K. Wang, M. Elkashlan, A. Nallanathan, J. Wang, and L. Hanzo, "Intelligent reflecting surface aided MIMO broadcasting for simultaneous wireless information and power transfer," IEEE J. Sel. Areas Commun., to appear.

[7] Y. Zeng, B. Clerckx, and R. Zhang, "Communications and signals design for wireless power transmission," IEEE Trans. Commun., vol. 65, no. 5, pp. 2264-2290, May 2017.

[8] J. D. Griffin and G. D. Durgin, "Gains for RF tags using multiple antennas," IEEE Trans. Antennas Propagat., vol. 56, no. 2, pp. 563-570, Feb. 2008. 